\title{
Clinicopathological and prognostic significance of circulating tumor cells in patients with head and neck cancer: a meta-analysis
}

This article was published in the following Dove Press journal:

OncoTargets and Therapy

4 August 2017

Number of times this article has been viewed

\author{
Taojiao Sun ${ }^{1, *}$ \\ Kun Zou ${ }^{2,3, *}$ \\ Zewei Yuan ${ }^{2}$ \\ Chaogang Yang ${ }^{2}$ \\ Xiaobin Lin ${ }^{2}$ \\ Bin Xiong ${ }^{2}$
}

'Department of Dental, ${ }^{2}$ Department of Oncology, Zhongnan Hospital of Wuhan University, Hubei

Key of Laboratory of Tumor Biological Behaviors and Hubei Cancer Clinical Study Center, ${ }^{3}$ Department of Oncology, Central Hospital of Wuhan, Wuhan, People's Republic of China

*These authors contributed equally to this work
Correspondence: Bin Xiong Department of Oncology, Zhongnan Hospital of Wuhan University, Hubei Key of Laboratory of Tumor Biological Behaviors and Hubei Cancer Clinical Study Center, Dong HU Road, 169, Wuhan 43007I, People's Republic of China

Email binxiong196I@whu.edu.cn
Background: Many studies have assessed the clinical use of circulating tumor cells (CTCs) in head and neck cancer, but the clinicopathological and prognostic significance of CTCs is still unclear. Materials and methods: Two authors systematically searched the studies independently with keywords in PubMed, MEDLINE, EMBASE, Science Citation Index Expanded and Cochrane Library (from inception to February 2017). The estimated hazard ratio (HR), risk ratio (RR) and their $95 \%$ confidence intervals (95\% CIs) were set as effect measures. All analyses were performed by STATA 12.0 .

Results: A total of 17 studies were included in this meta-analysis. Positive CTCs were significantly associated with poor overall survival ( $\mathrm{HR}=2.80,95 \% \mathrm{CI}: 1.34-5.86$ ), disease-free survival (HR $=3.86,95 \% \mathrm{CI}: 2.03-7.36)$ and progression-free survival $(\mathrm{HR}=3.31,95 \% \mathrm{CI}$ : 1.71-6.42). CTC-positive patients tend to have higher recurrence ( $\mathrm{RR}=2.13,95 \% \mathrm{CI}: 1.26-3.59)$ and regional lymph node metastasis $(\mathrm{RR}=1.18,95 \% \mathrm{CI}: 1.02-1.36)$ rate and a more advanced tumor stage ( $\mathrm{RR}=1.16,95 \% \mathrm{CI}: 1.03-1.32)$.

Conclusion: Our meta-analysis has confirmed the significant prognostic value of CTCs in head and neck cancer patients. The presence of CTCs could be used as a monitoring tool for tumor status of head and neck cancer, especially for the early detection of the tumor recurrence and progression, advanced disease and the node metastasis.

Keywords: head and neck cancer, circulating tumor cells, prognostic, clinicopathological characteristics, meta-analysis

\section{Background}

Head and neck squamous cell carcinoma (HNSCC) is a very common kind of epithelial cancer that accounts for the 7th most common cancer globally and arises from multiple anatomical sites such as oral cavity, oropharynx, hypopharynx and larynx. There are 500,000 new cases and 350,000 associated deaths for head and neck cancer in the world every year. About $60 \%$ of the new diagnostic patients of head and neck cancer are present with advanced disease (stage III/IV). ${ }^{1-3}$ Recently, multiple treatments were used for HNSCC, such as surgery, radiotherapy and cisplatin-based chemotherapy. But the 5-year survival rate for head and neck cancer patients is still $\sim 40 \%-50 \%$, and about $50 \%$ of the patients suffered local recurrence after the resection of the primary tumor and up to $25 \%$ developed distant metastases despite aggressive treatment. ${ }^{4,5}$ Therefore, early identification of metastasis and recurrence for the tumor progression and prognosis in patients with HNSCC is very important.

Circulating tumor cells (CTCs) are tumor cells that leave the primary tumor site and enter the bloodstream, where they can spread to other organs. In the past decades, many 
studies have shown that CTCs in the peripheral blood could be a valuable tool for real-time monitoring of tumor status, predicting potential metastasis and recurrence, monitoring treatment efficacy, determining drug-selection strategies and predicting the survival of cancer patients. ${ }^{6}$ Now, CTCs have been shown to have clinical associations with overall survival (OS) and response to therapy in many solid tumors, such as gastric cancer, lung cancer, breast cancer and colorectal cancer. ${ }^{6}$ But for the head and neck cancer, the clinical value is still unclear. In the previous studies, CTCs were only showed to predict more disease progression ${ }^{7}$ and a poor disease-free survival (DFS) ${ }^{8}$ in patients with head and neck cancer. In another study, although the prognostic value was confirmed, ${ }^{9}$ some insufficiency existed, all the included studies were published before 2014 and some CTC detection methods may be not suitable by using the monoclonal antibody E48 as CTC markers. And for the clinicopathological value of CTCs, all these studies failed to prove its value ${ }^{7-9}$ and only assessed the difference of CTCs positive rate. In recent years, some new studies were published with new sight of the value of CTCs for patients with head and neck cancer. ${ }^{10-12}$ Hence, the prognostic value and clinicopathological significance of CTCs are still controversial.

With the controversies existed in the clinicopathological and prognostic role of CTCs for head and neck cancer, here, we conducted the meta-analysis of published literature on this topic to summarize the evidence of the potential clinicopathological and prognostic value of CTCs in head and neck cancer.

\section{Materials and methods Search strategy}

Two authors (Sun and Zou) systematically searched the studies. With the keywords "head and neck cancer" or "head and neck squamous cell carcinoma", "circulating tumor cells" or "disseminated tumor cells" and "peripheral blood", we searched PubMed, EMBASE, Science Citation Index Expanded and Cochrane library (from inception to February 2017). An additional search through Google Scholar was conducted to identify other potentially relevant publications. Discrepancies were resolved by the third author (Yuan).

\section{Inclusion and exclusion criteria}

To keep our analysis accurate and reliable, the studies were selected according to the following inclusion and exclusion criteria. The inclusion criteria were 1) studies aiming at the association between the CTCs and OS, progression-free survival (PFS), DFS or the clinicopathological characteristics of head and neck cancer; 2) for OS, PFS and DFS, sufficient data to calculate hazard ratio (HR) and $95 \%$ confidence interval (95\% CI) being available; 3 ) original research published in only English language papers and 4) patients must be histologically confirmed with squamous cell carcinoma originating from head and neck. The exclusion criteria were 1) studies based on overlapping patients; 2) meta-analysis, review, case report, reporting of the expert experience and 3 ) outcome is not clear.

\section{Data extraction and outcomes}

Data retrieved from the studies included first author's name, year of publication, number of total and the CTC-positive patients, area of the patients, methods and markers for CTC detection, site and time of sample, follow-up duration of the study, recurrence and clinicopathological characteristics, prognostic value (OS, DFS or PFS), HR, etc. For studies with multiple blood sample time in CTC detection, only the results of baseline detection were used. If the HR and its 95\% CI for OS, DFS or PFS were not reported directly in the original study, we used the reported data to extrapolate the approximated HR by using software designed by Tierney et al. ${ }^{13}$ All data were extracted independently by two investigators (Sun and Zou), and disagreements were resolved by discussion.

\section{Statistical analysis}

We used the STATA 12.0 package (StataCorp, College Station, TX, USA) to analyze the data in our meta-analysis. The estimated risk ratio ( $R R$ ) was used to evaluate the correlation between CTCs and the tumor progression or clinicopathological characteristics; when RR $>1$, it means more frequency in the CTC-positive patients. The estimated HR was used to evaluate the prognostic effect (OS, DFS and PFS). All statistical values (HR and RR) were reported with 95\% CIs, and the two-sided $P$-value threshold for statistical significance was set at 0.05 . The Cochrane's $Q$ statistic and $I^{2}$ statistic were applied to evaluate the heterogeneity among studies. $P<0.1$ for the $Q$ statistic and/or $I^{2}>50 \%$ was considered significant heterogeneity, and the random-effects model was used, or the fixed-effects model was used conversely. ${ }^{14}$ We evaluated potential publication bias of the pooled data by Begg's test and Egger's test; $P<0.05$ was considered of significant publication bias. ${ }^{15}$ The Newcastle-Ottawa scale (NOS) was used to assess the quality of the retrieved studies, and scores 5-9 were of fair and scores 1-4 were of high bias. ${ }^{16}$ Moreover, we performed sensitivity analyses on prognostic value to explore whether the results were stable. This review follows the QUORUM and the Cochrane 
Collaboration guidelines (http://www.cochrane.de) for reporting meta-analysis (PRISMA statement). ${ }^{17}$

\section{Results}

\section{Baseline study characteristics and quality assessment}

\section{Literature search}

As shown in Figure 1, we initially searched 1,254 studies, of which 1,041 studies were excluded because of obviously not meeting the inclusion criteria. After reviewing the title and abstract, 171 relevant studies were excluded and 42 studies were available for reviewing full-text manuscript. Finally, 17 studies met our predefined inclusion criteria. ${ }^{10-12,18-31}$

Characteristics of the included studies are shown in Table 1. A total of 909 patients were identified. The number of patients in the individual studies ranged from 9 to 176 . The patients of the retrieved studies were from eight areas, and the retrieved studies were published from 2002 to 2016. Six studies exclusively focused on locally advanced stage (clinical stages III-IV). Except three studies, all the included studies mentioned the media follow-up duration that ranged from 6 to 60 months. For the quality assessment, the results are shown in Table 2. As all the studies were cohort studies, the NOS was used, most of the studies were of fair bias and four studies were of relatively high bias because of lack of assessment of the outcome and incomplete follow-up duration.

\section{Meta-analysis of clinicopathological characteristics}

Nine studies assessed the CTCs' status and tumor depth of infiltration. As shown in Figure 2A, a fixed model was used

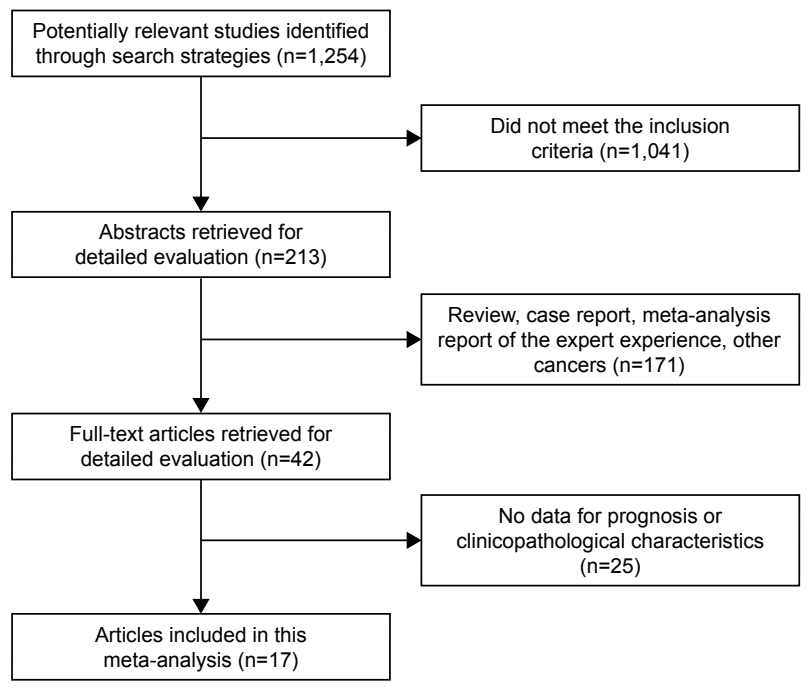

Figure I Selection of the included studies. without obvious sample heterogeneity $\left(I^{2}=39.8 \%, P=0.102\right)$. The pooled RR for tumor depth of infiltration was 1.20 (95\% CI: $0.99-1.45, P=0.058)$ and there was an edge significance between the CTC-positive and -negative HNSCC patients. The CTC-positive patients tend to deeper tumor infiltration (T3-T4) than the negative patients. A total of 11 studies assessed the CTCs' node status; the heterogeneity of these studies was of no significance $\left(I^{2}=34.2 \%, P=0.125\right)$, and the pooled RR was 1.18 (95\% CI: 1.02-1.36). Compared with the CTC-negative patients, patients with positive CTCs have higher regional lymph node metastasis rate $(P=0.021)$ (Figure 2B). Eight studies assessed the CTCs' status and tumor-node-metastasis (TNM) stage, with no heterogeneity $\left(I^{2}=0.0 \%, P=0.521\right)$. A fixed model was used, and the result showed that more advanced stage (III-IV) was in CTCpositive patients than CTC-negative patients $(\mathrm{RR}=1.16$, 95\% CI: 1.03-1.32) (Figure 2C).

For the correlation between CTCs and pathological grade, five studies were available. The fixed-effects model was used $\left(I^{2}=33.8 \%, P=0.196\right)$, and there was no significant difference between the CTC-positive and -negative patients ( $R R=1.02,95 \%$ CI: 0.98-1.06; Figure 2D). The same result was also found for the age and sex composition between CTC-positive and CTC-negative patients. The pooled RR for age composition was 1.05, 95\% CI: 0.75-1.46 (seven studies, $I^{2}=0.0 \%, P=0.606$; Figure $2 \mathrm{E}$ ). The pooled RR for sex composition was 1.04, 95\% CI: 0.88-1.24 (eight studies, $I^{2}=0.0 \%, P=0.606$; Figure $2 \mathrm{~F}$ ).

\section{Meta-analysis of prognostic value}

For the prognostic value, eight studies were available for OS, DFS or PFS. Of these studies, for the OS, five studies provided the calculated survival data or available Kaplan-Meier curves. With a relatively obvious heterogeneity $\left(I^{2}=57.6 \%\right.$, $P=0.051$ ), a random model was used and the pooled HR was 2.80 (95\% CI: 1.34-5.86; Figure 3A); a significant difference was found for OS between the CTC-positive and -negative HNSCC patients. For the DFS, four studies were available, with a significant heterogeneity $\left(I^{2}=74.4 \%, P=0.008\right)$; by using the random model, a significant HR for DFS was found ( $\mathrm{HR}=3.86,95 \% \mathrm{CI}: 2.03-7.36$; Figure 3B). Two eligible studies were pooled into the PFS meta-analysis; the pooled HR was $3.31,95 \%$ CI: $1.71-6.42$, without obvious heterogeneity $\left(I^{2}=0.0 \%, P=0.473\right.$; Figure $\left.3 \mathrm{C}\right)$.

At the same time, eight studies mentioned the data of tumor progression or recurrence. With a significant heterogeneity $\left(I^{2}=52.7 \%, P=0.039\right)$, a random model was used, the pooled RR was 2.13 (95\% CI: 1.26-3.59, $P=0.005$; 
Table I Basic characteristics of the retrieved studies

\begin{tabular}{|c|c|c|c|c|c|c|c|c|}
\hline Study & Year & $\begin{array}{l}\text { Number } \\
\text { CTC+/total }\end{array}$ & Area & $\begin{array}{l}\text { Tumor } \\
\text { stage }\end{array}$ & Sample site/time & Markers & Methods & $\begin{array}{l}\text { Follow-up } \\
\text { duration } \\
\text { (months) }\end{array}$ \\
\hline Li et al ${ }^{10}$ & 2016 & $20 / 38$ & China & I-IV & PB/before/Iw/Im TM & CD45 DAPI & Immunomagnetic & - \\
\hline Hsieh et al" & 2015 & $17 / 53$ & Taiwan & II-IV & PB/before TM & EpCAM/PDPN & $\begin{array}{l}\text { PowerMag } \\
\text { immunofluorescence }\end{array}$ & 10.5 \\
\hline Inhestern et $\mathrm{al}^{12}$ & 2015 & $32 / 40$ & Germany & III-IV & $\mathrm{PB} /$ before/during/after TM & EpCAM & Laser scanning cytometry & 24.7 \\
\hline Grisanti et $\mathrm{a}^{18}$ & 2014 & $14 / 53$ & Italy & III-IV & $\mathrm{PB} /$ before/during TM & EpCAM/CD45/DAPI & CellSearch & 25.0 \\
\hline Weller et al $^{19}$ & 2014 & $7 / 10$ & Germany & I-IV & PB/before TM & CK/CD45/DAPI & Immunofluorescence & 18 \\
\hline Tinhofer et $\mathrm{a}^{20}$ & 2014 & $42 / 144$ & Germany & I-IV & PB/before TM & EGFR mRNA & RT-PCR & 34 \\
\hline Grobe et $\mathrm{al}^{21}$ & 2014 & $10 / 80$ & Germany & I-IV & PB/before TM & EpCAM/CD45/DAPI & CellSearch & 30.1 \\
\hline Bozec et $\mathrm{al}^{22}$ & 2013 & $8 / 49$ & France & III-IV & PB/before TM & EpCAM/CD45/DAPI & CellSearch & 6 \\
\hline $\mathrm{He}$ et $\mathrm{a}^{23}$ & 2013 & $3 / 9$ & China & III-IV & PB/before TM & EpCAM/CD45/DAPI & CellSearch & 16 \\
\hline Buglione et $\mathrm{a}^{24}$ & 2012 & $11 / 73$ & Italy & I-IV & PB/before TM & EpCAM/CD 45/DAPI & CellSearch & 13.6 \\
\hline Nichols et $\mathrm{al}^{25}$ & 2012 & $6 / 15$ & Britain & III-IV & PB/before TM & EpCAM/CD 45/DAPI & CellSearch & 8 \\
\hline Hristozova et $\mathrm{a}^{26}$ & 2011 & $18 / 42$ & Germany & I-IV & PB/before TM & EpCAM, CK & Flow cytometry & - \\
\hline Jatana et $\mathrm{al}^{27}$ & 2010 & $34 / 48$ & USA & I-IV & PB/during TM & CK, CD45, DAPI & Immunocytochemistry & 38 \\
\hline Toyoshima et $\mathrm{a}^{28}$ & 2009 & $14 / 40$ & Germany & I-IV & $\mathrm{PB} /$ after TM & CK20 & RT-PCR & 43.5 \\
\hline Guney et $\mathrm{a}^{29}$ & 2007 & $7 / 21$ & Turkey & I-IV & PB/before TM & ЕрСAM & MACS & 36 \\
\hline Wollenberg et $\mathrm{al}^{30}$ & 2004 & $54 / 176$ & Germany & I-IV & BM/before TM & CKI9 & IHC-APAAP & 60 \\
\hline Wirtschafter et $\mathrm{al}^{3 !}$ & 2002 & $8 / 18$ & USA & I-IV & PB/before TM & CK20 & Immunocytochemistry & - \\
\hline
\end{tabular}

Abbreviations: CTC, circulating tumor cell; w, weeks; m, months; mRNA, messenger RNA; RT-PCR, reverse transcription-polymerase chain reaction; PB, peripheral blood; BM, bone marrow; IHC, immunohistochemistry; TM, treatment; DAPI, 4',6-diamidino-2-phenylindole; EpCAM, epithelial cell adhesion molecule; PDPN, podoplanin protein; CK, cytokeratin; EGFR, epidermal growth factor receptor; MACS, magnetic cell separation; APAAP, alkaline phosphatase-anti-alkaline phosphatase technique.

Figure 3D), a significant difference was found and the tumor progression or recurrence rate in CTC-positive HNSCC patients was 2.13 times of that in CTC-negative HNSCC patients. The CTC-positive patients before treatments tend to occur tumor recurrence after surgery or tumor progression after radiochemotherapy treatments.

\section{Sensitivity analysis and publication bias}

As limited studies mentioned the prognostic value of CTCs, only five for OS and four for DFS, and the heterogeneity for the prognostic value was relatively obvious, we did the sensitivity analysis for the pooled prognostic value, and the result is shown in Figure 4. For OS, DFS and tumor progression or recurrence, the pooled result showed no obvious changes after omitting any one of the involved studies. The results all showed that CTC-positive HNSCC patients have poor prognosis compared with the CTC-negative HNSCC patients. We confirmed that the result was stable and reliable.

Publication bias was detected by Begg's test and Egger's test. The result is shown in Table 3. No publication bias

Table 2 The assessment of the risk of bias in each cohort study using the NOS

\begin{tabular}{|c|c|c|c|c|c|c|c|c|c|c|}
\hline \multirow[t]{2}{*}{ Study } & \multicolumn{4}{|l|}{ Selection } & \multicolumn{2}{|c|}{ Comparability } & \multicolumn{3}{|l|}{ Outcome } & \multirow{2}{*}{ Star } \\
\hline & $\begin{array}{l}\text { Exposed } \\
\text { cohort }\end{array}$ & $\begin{array}{l}\text { Non-exposed } \\
\text { cohort }\end{array}$ & $\begin{array}{l}\text { Ascertainment } \\
\text { of exposure }\end{array}$ & $\begin{array}{l}\text { Demonstration } \\
\text { outcome }\end{array}$ & $\begin{array}{l}\text { Basic } \\
\text { factors }\end{array}$ & $\begin{array}{l}\text { Additional } \\
\text { factors }\end{array}$ & Assessment & $\begin{array}{l}\text { Follow- } \\
\text { up }\end{array}$ & Adequacy & \\
\hline Li et $a^{10}$ & 0 & I & I & I & I & 0 & 0 & 0 & 0 & 4 \\
\hline Hsieh et al' & I & I & I & I & 0 & 0 & I & I & 0 & 6 \\
\hline Inhestern et $\mathrm{al}^{12}$ & 0 & I & I & 1 & 0 & 0 & I & I & I & 6 \\
\hline Grisanti et al $^{18}$ & 0 & I & I & 0 & I & 0 & I & I & I & 6 \\
\hline Weller et al ${ }^{19}$ & I & I & I & 0 & 0 & 0 & I & I & 0 & 5 \\
\hline Tinhofer et $\mathrm{al}^{20}$ & I & I & I & 0 & I & 0 & I & I & 0 & 6 \\
\hline Grobe et $\mathrm{al}^{2 \mathrm{I}}$ & I & I & I & I & 0 & 0 & I & I & I & 7 \\
\hline Bozec et $\mathrm{al}^{22}$ & 0 & 1 & I & I & I & 0 & 0 & 0 & 0 & 4 \\
\hline He et $\mathrm{al}^{23}$ & 0 & I & I & I & 0 & 0 & 1 & 0 & 0 & 4 \\
\hline Buglione et $\mathrm{al}^{24}$ & I & I & I & 1 & I & 0 & I & 0 & 0 & 6 \\
\hline Nichols et al ${ }^{25}$ & 0 & I & I & 1 & 0 & 0 & 1 & 0 & 0 & 4 \\
\hline Hristozova et $\mathrm{al}^{26}$ & I & I & I & 1 & I & 0 & 0 & 0 & 0 & 5 \\
\hline Jatana et $\mathrm{al}^{27}$ & I & I & I & 0 & 0 & 0 & I & I & I & 6 \\
\hline Toyoshima et $\mathrm{al}^{28}$ & I & I & I & 0 & I & 0 & I & I & I & 7 \\
\hline Guney et $\mathrm{al}^{29}$ & 0 & I & I & I & I & 0 & I & 0 & 0 & 5 \\
\hline Wollenberg et $\mathrm{al}^{30}$ & I & I & I & I & I & 0 & I & 0 & 0 & 6 \\
\hline Wirtschafter et $\mathrm{al}^{31}$ & I & I & I & 0 & I & 0 & 0 & 0 & 0 & 4 \\
\hline
\end{tabular}

Abbreviation: NOS, Newcastle-Ottawa scale. 

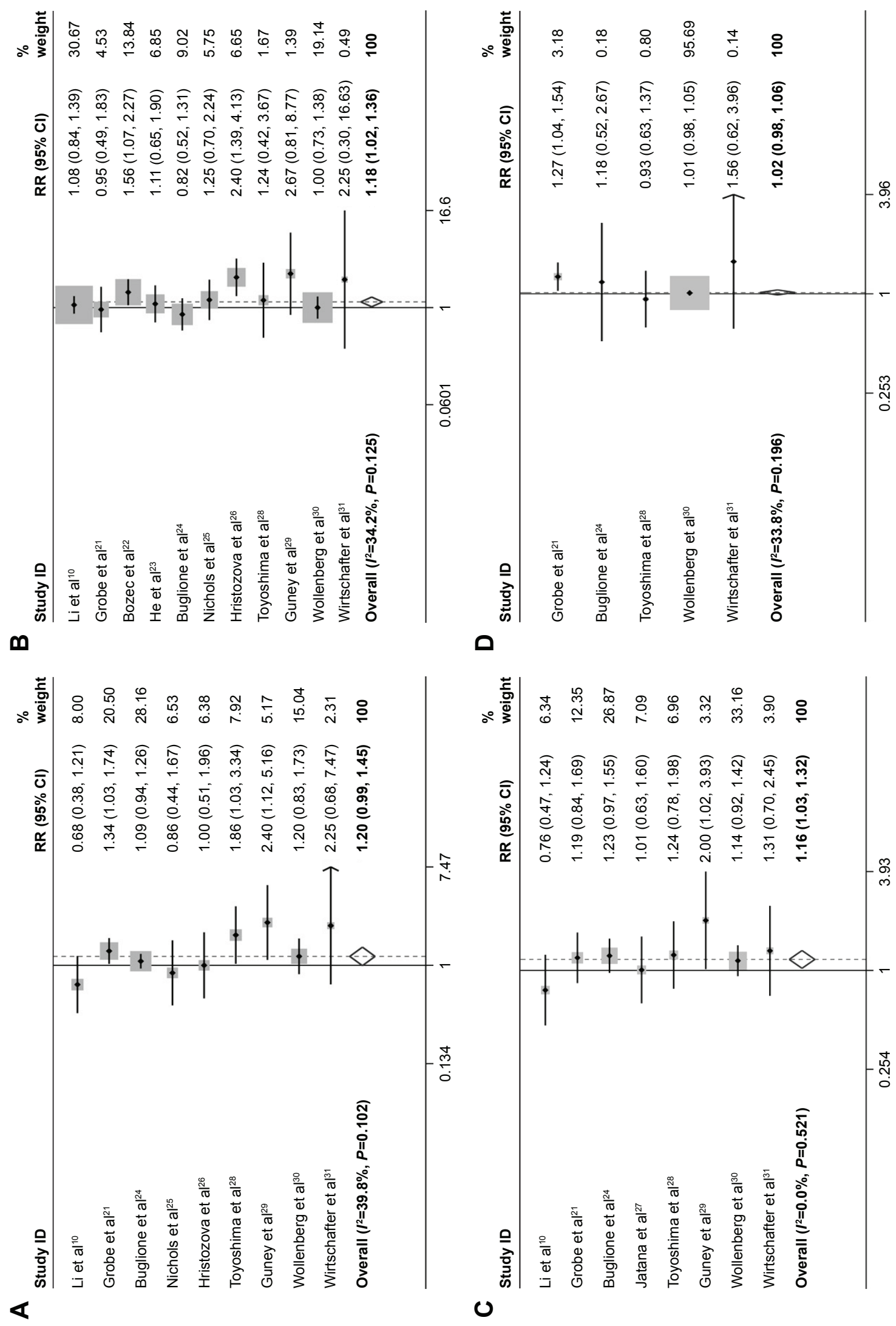

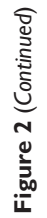




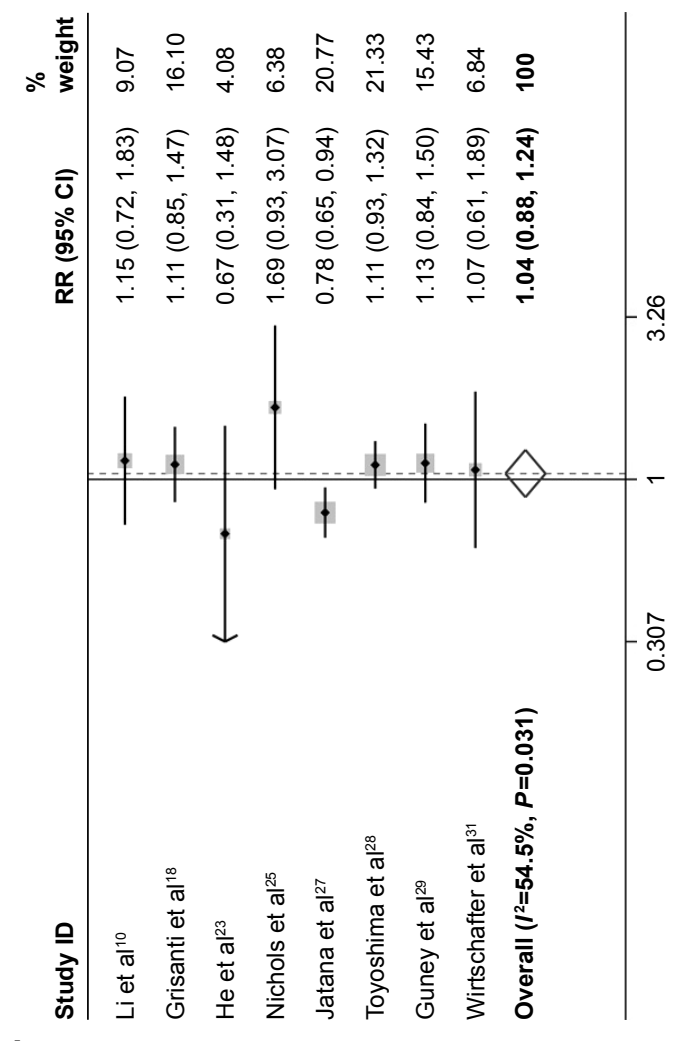

丩

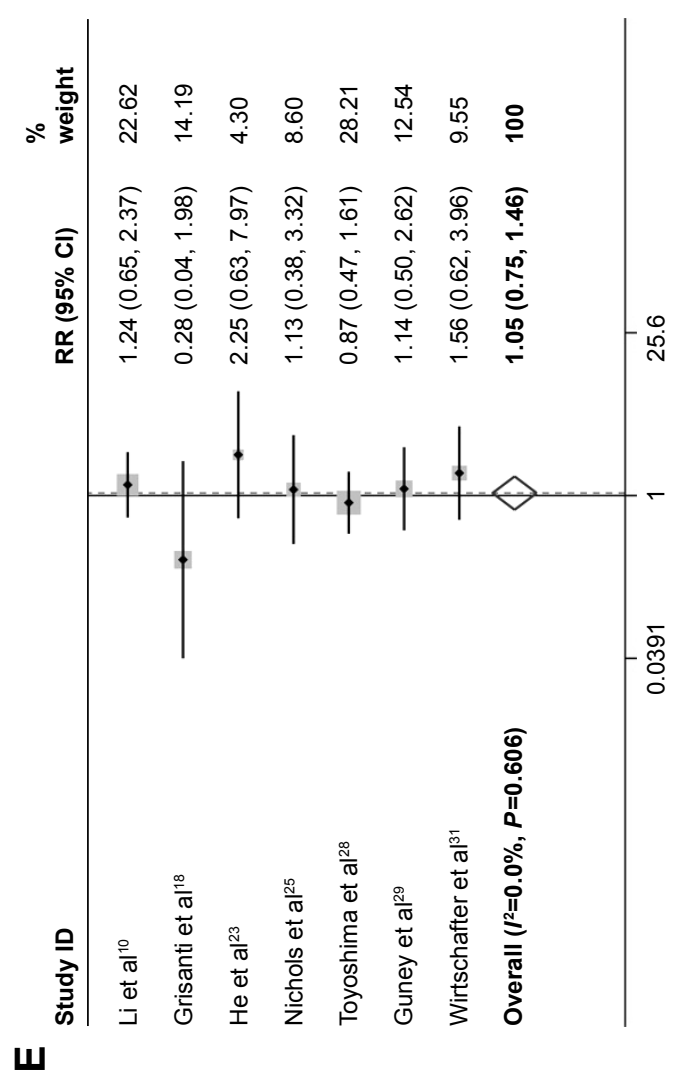

was found in all the variables we analyzed in this metaanalysis.

\section{Discussion}

Head and neck cancer is a very common malignant disease with high morbidity and mortality. Despite the development of the diagnostic method and use of aggressive multiple therapeutic regimens, most of the HNSCC patients finally develop locoregional recurrence or metastatic disease, and the 5 -year survival rate is $~ 50 \%{ }^{4,32}$ So it is very important to find a reliable blood marker to monitor locoregional or distant recurrence and predict the prognosis of the HNSCC patients. Recently, the research on CTCs indicates that CTCs may act as very significant prognostic markers in patients with HNSCC. ${ }^{12}$ In this meta-analysis, by summarizing all relevant studies, we first confirmed the clinicopathological and prognostic significance of CTCs in patients with head and neck cancer comprehensively. In this meta-analysis, we paid more attention to the clinical application value of CTC detection, so we divided the patients into CTCpositive and CTC-negative HNSCC when assessing the correlation between CTC detection and clinicopathological characteristics. And we assessed the difference of common clinicopathological characteristics between the CTC-positive and CTC-negative HNSCC patients, then we first confirmed that CTC-positive patients were tend to have more advanced stage (III-IV) and higher regional lymph node metastasis rate than the CTC-negative patients. This is different from the other meta-analysis, ${ }^{7-9}$ as they assessed the difference of CTC-positive rate between patients with different clinical characteristics.

In this meta-analysis, we made a comprehensive analysis on the prognostic value of CTCs and provided strong evidence that positive CTCs were significantly associated with poor OS, DFS and PFS of head and neck cancer patients. Positive CTCs were a poor prognosis for patients with head and neck cancer. For OS, we first found that the CTC-positive patients had a risk increase of death by 1.8 times of CTC-negative patients (pooled HR $=2.80 ; 95 \%$ CI: 1.34-5.86). For DFS, the risk of tumor progression or relative death for CTC-positive patients was 3.86 times of that for CTC-negative patients (pooled HR $=3.86,95 \%$ CI: 2.03-7.36), and the result was also confirmed in other studies. For the PFS, we confirmed that CTC-positive patients tend to easier to tumor progression than the CTCnegative patients. More death and tumor progression were found in CTC-positive patients, compared with CTCnegative patients. Besides, we also found the CTC-positive HNSCC patients have a higher recurrence rate than negative 

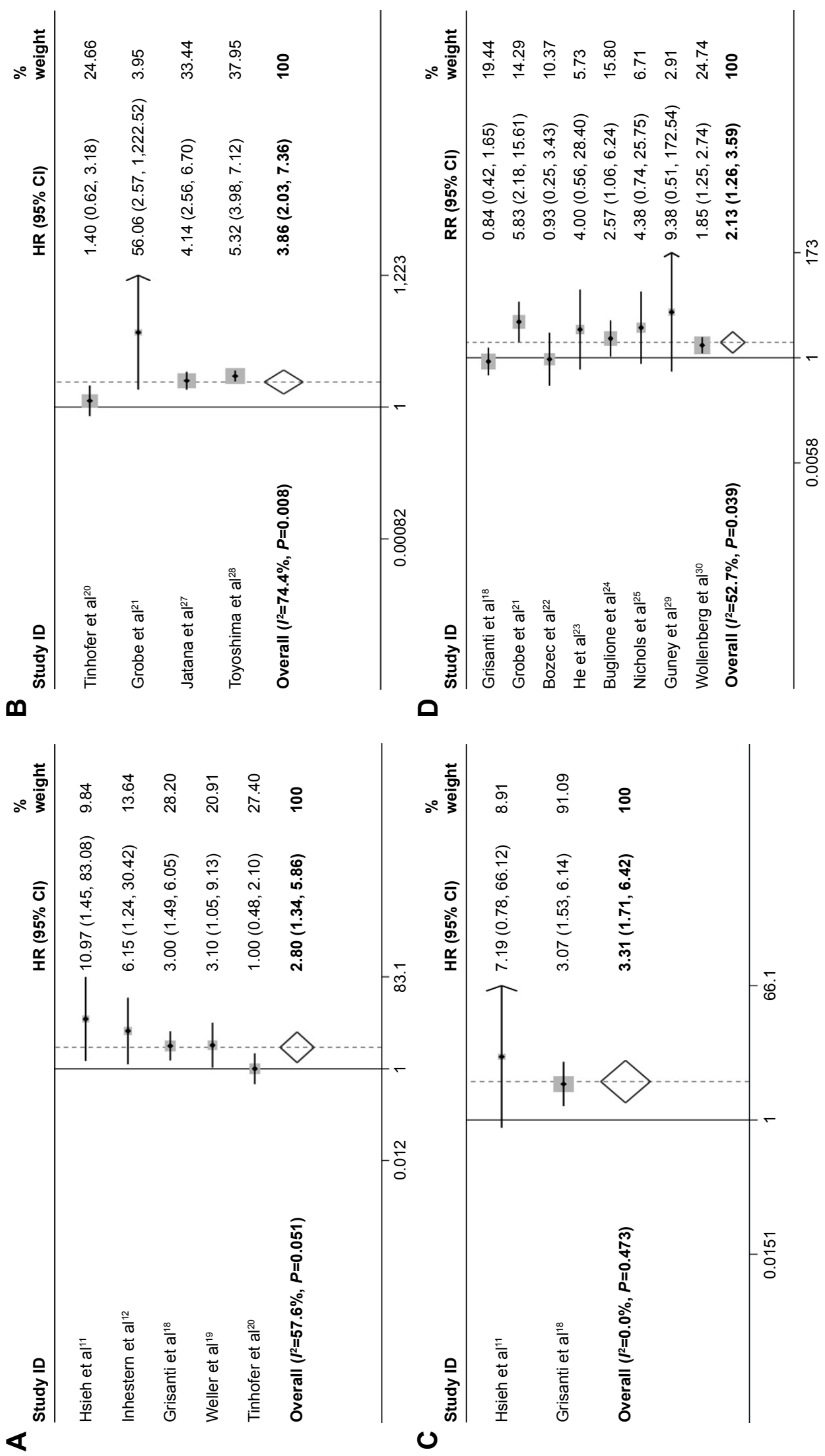
A
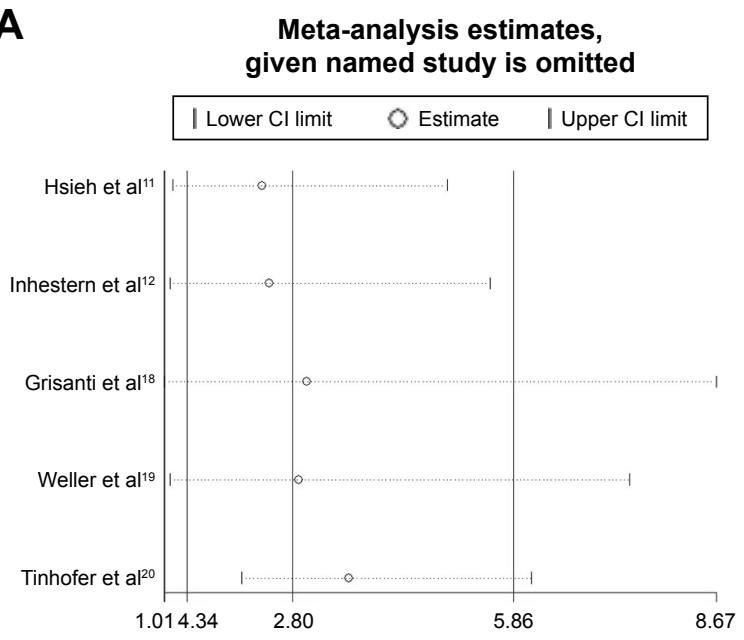

B
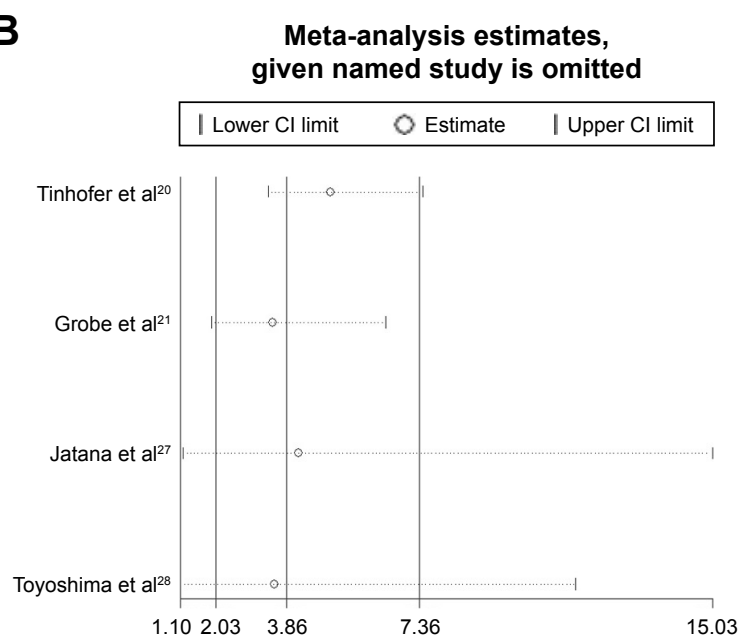

C Meta-analysis estimates, given named study is omitted

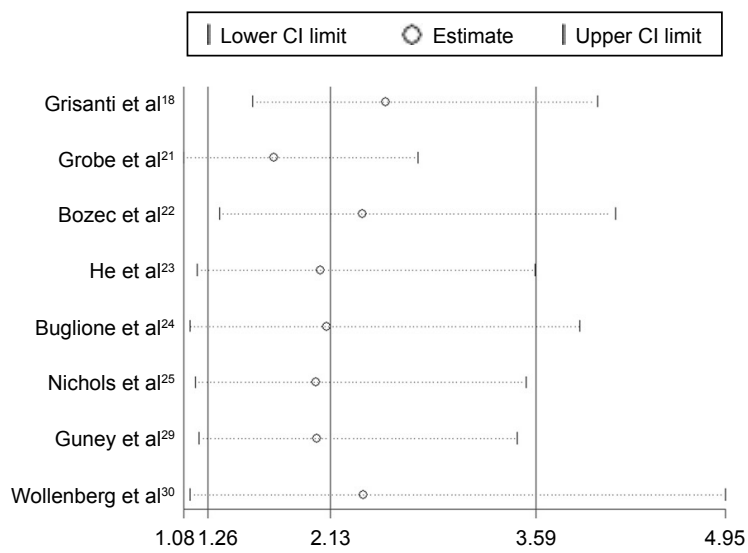

Figure 4 Sensitivity analysis for the pooled prognostic value, (A) OS, (B) DFS, and (C) recurrence.

Abbreviations: OS, overall survival; DFS, disease-free survival; $\mathrm{Cl}$, confidence interval.

patients. The CTC-positive patients were easier to occur tumor recurrence or progression after treatments. After escaped from primary tumor and shed into blood, CTCs could be activated due to the loss of biological control by

Table 3 Publication bias by Egger's and Begg's test

\begin{tabular}{lll}
\hline Variables & \multicolumn{1}{c}{$\boldsymbol{P}$-value } & \\
\cline { 2 - 3 } & Egger's & Begg's \\
\hline Age & 0.849 & $>0.999$ \\
Sex & 0.514 & 0.711 \\
Tumor infiltration & 0.45 & 0.348 \\
Node metastasis & 0.252 & 0.213 \\
TNM stage & 0.825 & 0.536 \\
Pathological grade & 0.309 & 0.462 \\
Recurrence & 0.328 & 0.174 \\
PFS & - & $>0.999$ \\
DFS & 0.862 & 0.734 \\
OS & 0.053 & 0.089 \\
\hline Abreviation
\end{tabular}

Abbreviations: TNM, tumor-node-metastasis; PFS, progression-free survival; DFS, disease-free survival; OS, overall survival. primary tumor and the alteration of internal environment, and then the CTCs can form the new metastasis or recurrence. ${ }^{33}$ So the detection of CTCs in patients with head and neck cancer can predict the prognosis and find the patients who have high recurrence and tumor progression rate, and in these cases, we can give these patients early and aggressive treatment.

As shown in our meta-analysis, limited studies mentioned the prognostic value of CTCs, only five for OS and four for DFS, and the heterogeneity for the prognostic value was relatively obvious. Thinking that the result may be affected by single studies or the accidental factor, we did the sensitive analysis for the pooled prognostic value to test the reliability and stability of our conclusion. Finally, we found that the result did not change obviously in the sensitive analysis. So we confirm the conclusion that positive CTCs in patients with head and neck cancer can predict the poor prognosis is stable and reliable. 
Moreover, we assessed the difference of common clinicopathological characteristics between the CTC-positive and CTC-negative HNSCC patients. Our meta-analysis indicated that positive CTCs were significantly associated with TNM staging and regional lymph node metastasis. The CTC-positive patients were tend to have more advanced stage (III-IV) and with higher regional lymph node metastasis rate than the CTC-negative patients. But for the tumor depth of infiltration, there was no significant difference between CTC-positive and -negative patients; this may be because the CTCs were tend to represent the metastatic ability of tumor rather than local invasion. ${ }^{34}$ We also found that the CTCs have no correlation with the age and sex composition of the HNSCC patients. As detection of CTCs is very convenient and comfortable for patients and is repeatable in a noninvasive manner, the presence of CTCs could be used as a monitoring tool for tumor status of head and neck cancer, especially for the early detection of the advanced disease and the node metastasis. The detection of CTCs in head and neck cancer patients can early identify those patients who were with advanced or metastasis disease. Thus, we can give these patients more aggressive treatments.

The positive CTCs may also indicate the poor response of the chemotherapy treatment for the patients with head and neck cancer. Grisanti et al ${ }^{18}$ showed that for the HNSCC patients ( 45 patients) treated with chemotherapy, the clinical disease control rate was $45 \%$ in patients with CTCs negative (15/33), while for the 12 patients with positive CTCs at baseline, the clinical disease control rate was $8 \%$, a significant difference was found $(P=0.03)$. Buglione et $\mathrm{al}^{24}$ also showed that the CTCs positive rate was obviously lower in patients with complete response than without complete response after chemotherapy or radiochemotherapy ( $9 \%$ vs $24 \%)$. So the CTCs may be a very useful tool to assess the efficiency of the non-surgery treatment.

There were some limitations in our meta-analysis. In this meta-analysis, we used the extracted data and not the original data; limited studies were used for the prognostic value, and the heterogeneity was relatively obvious. The result may be influenced by accidental factor; multiple CTC detection methods were involved in our meta-analysis, and the time and sit of the sample exists difference, these all may contribute to the heterogeneity of our meta-analysis; efficiency of non-surgery treatment could not be analyzed due to the lack of relative data. Therefore, large-scale multicenter studies in homogeneous patients were needed to explore the prognostic value of CTCs.

\section{Conclusion}

Our meta-analysis confirmed the clinicopathological and prognostic significance of CTCs in patients with head and neck cancer comprehensively. CTC detection has great potential clinical application in head and neck cancer. Positive CTCs in patients with head and neck cancer can predict the poor prognosis and the high recurrence and tumor progression rate. The CTC-positive patients tend to have more advanced stage (III-IV) and higher regional lymph node metastasis rate. The presence of CTCs could be used as a monitoring tool for tumor status of head and neck cancer, especially for the early detection of the advanced disease and the node metastasis. In the future, large-scale multicenter studies by using the same standardized detection platforms are needed to reduce the inconsistencies across studies.

\section{Disclosure}

The authors report no conflicts of interest in this work.

\section{References}

1. Kang H, Kiess A, Chung CH. Emerging biomarkers in head and neck cancer in the era of genomics. Nat Rev Clin Oncol. 2015;12(1):11-26.

2. Torre LA, Bray F, Siegel RL, Ferlay J, Lortet-Tieulent J, Jemal A. Global cancer statistics, 2012. CA Cancer J Clin. 2015;65(2):87-108.

3. Marur S, Forastiere AA. Head and neck squamous cell carcinoma: update on epidemiology, diagnosis, and treatment. Mayo Clin Proc. 2016;91(3):386-396.

4. Boussios S, Seraj E, Zarkavelis G, et al. Management of patients with recurrent/advanced cervical cancer beyond first line platinum regimens: where do we stand? A literature review. Crit Rev Oncol Hematol. 2016; 108:164-174.

5. Peng PJ, Lv BJ, Wang ZH, et al. Multi-institutional prospective study of nedaplatin plus S-1 chemotherapy in recurrent and metastatic nasopharyngeal carcinoma patients after failure of platinum-containing regimens. Ther Adv Med Oncol. 2017;9(2):68-74.

6. Lianidou ES, Strati A, Markou A. Circulating tumor cells as promising novel biomarkers in solid cancers. Crit Rev Clin Lab Sci. 2014;51(3): $160-171$.

7. Wang Z, Cui K, Xue Y, Tong F, Li S. Prognostic value of circulating tumor cells in patients with squamous cell carcinoma of the head and neck: a systematic review and meta-analysis. Med Oncol. 2015; 32(5): 164 .

8. Wu XL, Tu Q, Faure G, Gallet P, Kohler C, Bittencourt MC. Diagnostic and prognostic value of circulating tumor cells in head and neck squamous cell carcinoma: a systematic review and meta-analysis. Sci Rep. 2016;6:20210.

9. Chen R, Zhou Y, Wen B. Clinicopathological and prognostic significance of circulating tumor cells in patients with head and neck cancer: a meta-analysis. Int J Med Phys Clin Eng Radiat Oncol. 2016; 05(2):138-149.

10. Li F, Liu J, Song D, Zhang Q, Ding N, He X. Circulating tumor cells in the blood of poorly differentiated nasal squamous cell carcinoma patients: correlation with treatment response. Acta Otolaryngol. 2016; 136(11):1164-1167.

11. Hsieh JC, Lin HC, Huang CY, et al. Prognostic value of circulating tumor cells with podoplanin expression in patients with locally advanced or metastatic head and neck squamous cell carcinoma. Head Neck. 2015;37(10):1448-1455. 
12. Inhestern J, Oertel K, Stemmann V, et al. Prognostic role of circulating tumor cells during induction chemotherapy followed by curative surgery combined with postoperative radiotherapy in patients with locally advanced oral and oropharyngeal squamous cell cancer. PLoS One. 2015;10(7):e132901.

13. Tierney JF, Stewart LA, Ghersi D, Burdett S, Sydes MR. Practical methods for incorporating summary time-to-event data into meta-analysis. Trials. 2007;8:16.

14. Asimit J, Day-Williams A, Zgaga L, Rudan I, Boraska V, Zeggini E. An evaluation of different meta-analysis approaches in the presence of allelic heterogeneity. Eur J Hum Genet. 2012;20(6):709-712.

15. Papageorgiou SN, Papadopoulos MA, Athanasiou AE. Assessing small study effects and publication bias in orthodontic meta-analyses: a metaepidemiological study. Clin Oral Investig. 2014;18(4):1031-1044.

16. Stang A. Critical evaluation of the Newcastle-Ottawa scale for the assessment of the quality of nonrandomized studies in meta-analyses. Eur J Epidemiol. 2010;25(9):603-605.

17. Panic N, Leoncini E, de Belvis G, Ricciardi W, Boccia S. Evaluation of the endorsement of the preferred reporting items for systematic reviews and meta-analysis (PRISMA) statement on the quality of published systematic review and meta-analyses. PLoS One. 2013;8(12):e83138.

18. Grisanti S, Almici C, Consoli F, et al. Circulating tumor cells in patients with recurrent or metastatic head and neck carcinoma: prognostic and predictive significance. PLoS One. 2014;9(8):e103918.

19. Weller P, Nel I, Hassenkamp P, et al. Detection of circulating tumor cell subpopulations in patients with head and neck squamous cell carcinoma (HNSCC). PLoS One. 2014;9(12):e113706.

20. Tinhofer I, Konschak R, Stromberger C, et al. Detection of circulating tumor cells for prediction of recurrence after adjuvant chemoradiation in locally advanced squamous cell carcinoma of the head and neck. Ann Oncol. 2014;25(10):2042-2047.

21. Grobe A, Blessmann M, Hanken H, et al. Prognostic relevance of circulating tumor cells in blood and disseminated tumor cells in bone marrow of patients with squamous cell carcinoma of the oral cavity. Clin Cancer Res. 2014;20(2):425-433.

22. Bozec A, Ilie M, Dassonville O, et al. Significance of circulating tumor cell detection using the CellSearch system in patients with locally advanced head and neck squamous cell carcinoma. Eur Arch Otorhinolaryngol. 2013;270(10):2745-2749.

23. He S, Li P, He S, et al. Detection of circulating tumour cells with the CellSearch system in patients with advanced-stage head and neck cancer: preliminary results. J Laryngol Otol. 2013;127(8):788-793.
24. Buglione M, Grisanti S, Almici C, et al. Circulating tumour cells in locally advanced head and neck cancer: preliminary report about their possible role in predicting response to non-surgical treatment and survival. Eur J Cancer. 2012;48(16):3019-3026.

25. Nichols AC, Lowes LE, Szeto CC, et al. Detection of circulating tumor cells in advanced head and neck cancer using the CellSearch system. Head Neck. 2012;34(10):1440-1444.

26. Hristozova T, Konschak R, Stromberger C, et al. The presence of circulating tumor cells (CTCs) correlates with lymph node metastasis in nonresectable squamous cell carcinoma of the head and neck region (SCCHN). Ann Oncol. 2011;22(8):1878-1885.

27. Jatana KR, Balasubramanian P, Lang JC, et al. Significance of circulating tumor cells in patients with squamous cell carcinoma of the head and neck: initial results. Arch Otolaryngol Head Neck Surg. 2010; 136(12):1274-1279.

28. Toyoshima T, Vairaktaris E, Nkenke E, Schlegel KA, Neukam FW, Ries J. Hematogenous cytokeratin 20 mRNA detection has prognostic impact in oral squamous cell carcinoma: preliminary results. Anticancer Res. 2009;29(1):291-297.

29. Guney K, Yoldas B, Ozbilim G, Derin AT, Sarihan S, Balkan E. Detection of micrometastatic tumor cells in head and neck squamous cell carcinoma. A possible predictor of recurrences? Saudi Med J. 2007; 28(2):216-220.

30. Wollenberg B, Walz A, Kolbow K, Pauli C, Chaubal S, Andratschke M. Clinical relevance of circulating tumour cells in the bone marrow of patients with SCCHN. Onkologie. 2004;27(4):358-362.

31. Wirtschafter A, BenningerMS, Moss TJ,UmielT, BlazoffK, Worsham MJ. Micrometastatic tumor detection in patients with head and neck cancer: a preliminary report. Arch Otolaryngol Head Neck Surg. 2002; 128(1):40-43.

32. Yom SS, Ganti AK, Dietz A. What's new in head and neck cancer: key findings in 2015-2016 from ECCO/ESMO, ASTRO, and the multidisciplinary head and neck cancer symposium. Am Soc Clin Oncol Educ Book. 2016;35:176-183.

33. Mohme M, Riethdorf S, Pantel K. Circulating and disseminated tumour cells - mechanisms of immune surveillance and escape. Nat Rev Clin Oncol. 2017;14(3):155-167.

34. Dasgupta A, Lim AR, Ghajar CM. Circulating and disseminated tumor cells: harbingers or initiators of metastasis? Mol Oncol. 2017; 11(1):40-61.
OncoTargets and Therapy

\section{Publish your work in this journal}

OncoTargets and Therapy is an international, peer-reviewed, open access journal focusing on the pathological basis of all cancers, potential targets for therapy and treatment protocols employed to improve the management of cancer patients. The journal also focuses on the impact of management programs and new therapeutic agents and protocols on

\section{Dovepress}

patient perspectives such as quality of life, adherence and satisfaction The manuscript management system is completely online and includes a very quick and fair peer-review system, which is all easy to use. Visit http://www.dovepress.com/testimonials.php to read real quotes from published authors. 